(c) 2021 Universidad Nacional Autónoma de México, Facultad de Estudios Superiores Zaragoza.

Este es un artículo Open Access bajo la licencia CC BY-NC-ND (http://creativecommons.org/licenses/by-nc-nd/4.0/).

TIP Revista Especializada en Ciencias Químico-Biológicas, 24: 1-10, 2021.

https://doi.org/10.22201/fesz.23958723e.2021.384

\title{
Iconella guatimalensis (Ehrenberg) Ruck \& Nakov (Bacillariophyta) como especie indicadora de condiciones de nivel lacustre bajo en tres registros sedimentarios lacustres del centro de México
}

\author{
Maripili Ramírez-Nava ${ }^{1 *}$, Margarita Caballero² y Mercedes Vázquez ${ }^{2,3}$ \\ ${ }^{1}$ Posgrado en Ciencias del Mar y Limnología, ${ }^{2}$ Laboratorio de Paleolimnología, Instituto de Geofísica, \\ ${ }^{3}$ Facultad de Ciencias, Universidad Nacional Autónoma de México; Av. Ciudad Universitaria, \\ C.P. 04510, Coyoacán, Ciudad de México, México. E-mail: *diatopily@yahoo.com.mx
}

\begin{abstract}
RESUMEN
Iconella guatimalensis es una diatomea dulceacuícola y de gran tamaño, inicialmente descrita a partir de un material proveniente de Guatemala y reportada en diversas localidades del Neotrópico. En México, sin embargo, únicamente ha sido confirmada en dos localidades modernas. En este trabajo verificamos la presencia de esta especie con baja abundancia $(<10 \%)$ en los registros sedimentarios del Holoceno de los lagos Santa María del Oro (SMO) y Coatetelco (COA). Se presenta una descripción morfológica basada en la observación de varios ejemplares en microscopía de luz y electrónica de barrido. La distribución de esta especie en los dos lagos coincide con ventanas de tiempo que se han identificado como de ambientes de aguas más someras y climas secos (Sequía del Clásico Tardío, 600-1000 d.C. y de la Pequeña Edad de Hielo, 1315-1860 d.C.). La distribución de I. guatimalensis en una de las secuencias estratigráficas de lago SMO coincide con incrementos en la abundancia de otras especies de diatomeas de habito bentónico, lo que corrobora que se trata de periodos con un nivel lacustre bajo. Los lapsos de tiempo en los que esta especie muestra su máxima abundancia en los dos registros combinados fueron: 3100-2300 a.C. (5050-4260 A.P.); 1040-580 a.C. (3000-2530 A.P.); 50-900 d.C. (1900-1050 A.P.); y 1315-1860 d.C. (635-90 A.P.).
\end{abstract}

Palabras Clave: diatomeas continentales, Surirellales, paleoindicador, registro paleolimnológico, lago.

\section{Iconella guatimalensis (Ehrenberg) Ruck \& Nakov (Bacillariophyta) as an indicator species} for low lake level conditions in three lake sedimentary records from central Mexico

\begin{abstract}
Iconella guatimalensis is a large freshwater diatom, initially described in material from Guatemala and reported in various locations in the Neotropics. However, it has only been confirmed in two locations for modern environments in Mexico. In this paper we report the presence of this species in low abundances $(<10 \%)$ in the Holocene sedimentary records of lakes Santa María del Oro (SMO) and Coatetelco (COA). A morphological description was integrated from observations of several specimens in light and scanning electron microscopy. The distribution of this species in the two lakes coincides with time windows that have been identified as shallow-water environments and dry climates (Late Classic Drought A.D. 600-1000 and the Little Ice Age, A.D. 1315-1860). The distribution of I. guatimalensis also agrees with increases in the abundances of other benthic diatoms in SMO, corroborating that it can be considered as an indicator of periods with low lake levels. The times of maximum abundances of this species in the two combined records were: B.C. $3100-2300$ (5050-4260 B.P.); B.C. 1040-580 (3000-2530 B.P.); A.D. 50-900 (1900-1050 B.P.); y A.D. $1315-1860$ (635-90 B.P.).
\end{abstract}

Keywords: continental diatoms, Surirellales, paleoindicator, paleolimnological record, lake.

Artículo recibido el 30 de marzo del 2021.

Artículo aceptado el 07 de noviembre del 2021. 


\section{INTRODUCCIÓN}

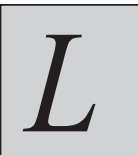

as diatomeas, pertenecientes a la división Bacillariophyta, son algas unicelulares eucariontes ( 5 a 2,000 $\mu \mathrm{m}$ de longitud) que pueden vivir flotando en forma libre o adheridas sobre diversos sustratos.

Estas algas microscópicas se caracterizan, entre otras cosas, por tener una pared celular de dióxido de silicio $\left(\mathrm{SiO}_{2}\right)$, a la que se denomina frústulo, formada por tres componentes morfológicos fundamentales, dos piezas principales denominadas valvas y un conjunto de bandas conectivas denominado cíngulo (Round, Crawford \& Mann, 1990). Las diatomeas son excelentes bioindicadores, debido a que la distribución de sus especies se encuentra directamente relacionada con variables ambientales como la salinidad, el $\mathrm{pH}$, los niveles de nutrientes, la profundidad del cuerpo de agua, etcétera. (Sapelko, Pozdnyakov, Kuznetsov \& Ludikova, 2019). Por ejemplo, especies comunes en el centro de México como Nitzschia frustulum o Cyclotella meneghiniana, son indicadoras de ambientes lacustres salobres (Caballero, Lozano-García, Ortega-Guerrero \& Correa-Metrio, 2019), mientas que otras como Stephanodiscus niagarae no toleran una elevada salinidad, pero son en cambio indicadoras de niveles de nutrientes elevados y tienen una afinidad por condiciones climáticas templadas (Avendaño, Caballero \& Vázquez, 2021). Cuando las diatomeas mueren, sus frústulos se acumulan y preservan en los sedimentos por miles de años debido a que son resistentes a la degradación, formando un excelente archivo paleoambiental.

La taxonomía de las diatomeas se basa en gran media en la morfología de los frústulos, la simetría y patrones de ornamentación de las valvas hacen posible la identificación taxonómica de poblaciones a nivel de especie e inclusive de variedad. Una de las estructuras más importantes para la taxonomía de las diatomeas es la presencia de una fisura longitudinal denominada sistema del rafe, que es característica de la subclase Bacillariophycidae. El sistema del rafe es una estructura que permite el desplazamiento de los frústulos sobre un sustrato; morfológicamente consiste en dos fisuras longitudinales, generalmente localizadas a lo largo del eje principal de simetría bilateral, denominado eje apical. Las dos fisuras o ramas del rafe se encuentran en el centro de la cara valvar, formando el nódulo central, mientras que, hacia el extremo de la valva, las ramas del rafe terminan en los nódulos polares (Figura 1A). El sistema del rafe puede tener diversas modificaciones sobre este patrón general, puede desplazarse hacia el margen valvar, y además localizarse en un canal ubicado sobre la superficie del margen valvar o el canal puede elevarse formando una estructura denominada quilla (Figura 1B). El canal del rafe mantiene comunicación con el interior de la célula a través de poros simples (portulae), y cuando se encuentra elevado estos poros simples se modifican a elementos de soporte altamente silicificados denominados fibulae que alternan con aberturas o fenestrae.
El orden Surirellales es uno de los dos órdenes dentro de la subclase Bacillariophycidae que se caracterizan por la presencia de un canal del rafe. Este orden fue recientemente reclasificado a partir de caracteres morfológicos, moleculares, filogenéticos y ecológicos (Ruck, Nakov, Alverson \& Theriot, 2016a), incorporando a todos los géneros que antes se ubicaban dentro del orden Rhopalodiales. Dentro de este nuevo concepto de Surirellales se incluyen todas las especies que han desarrollado una disposición del canal del rafe en la periferia del margen valvar (circunferencial), asociado con un cambio en la geometría de la valva en donde el eje de simetría transapical tiene un mayor desarrollo que el eje apical (Figura 1C), por lo que en los extremos opuestos de la valva se ubican, por un lado el nódulo central (extremos proximales) y en el opuesto los nódulos

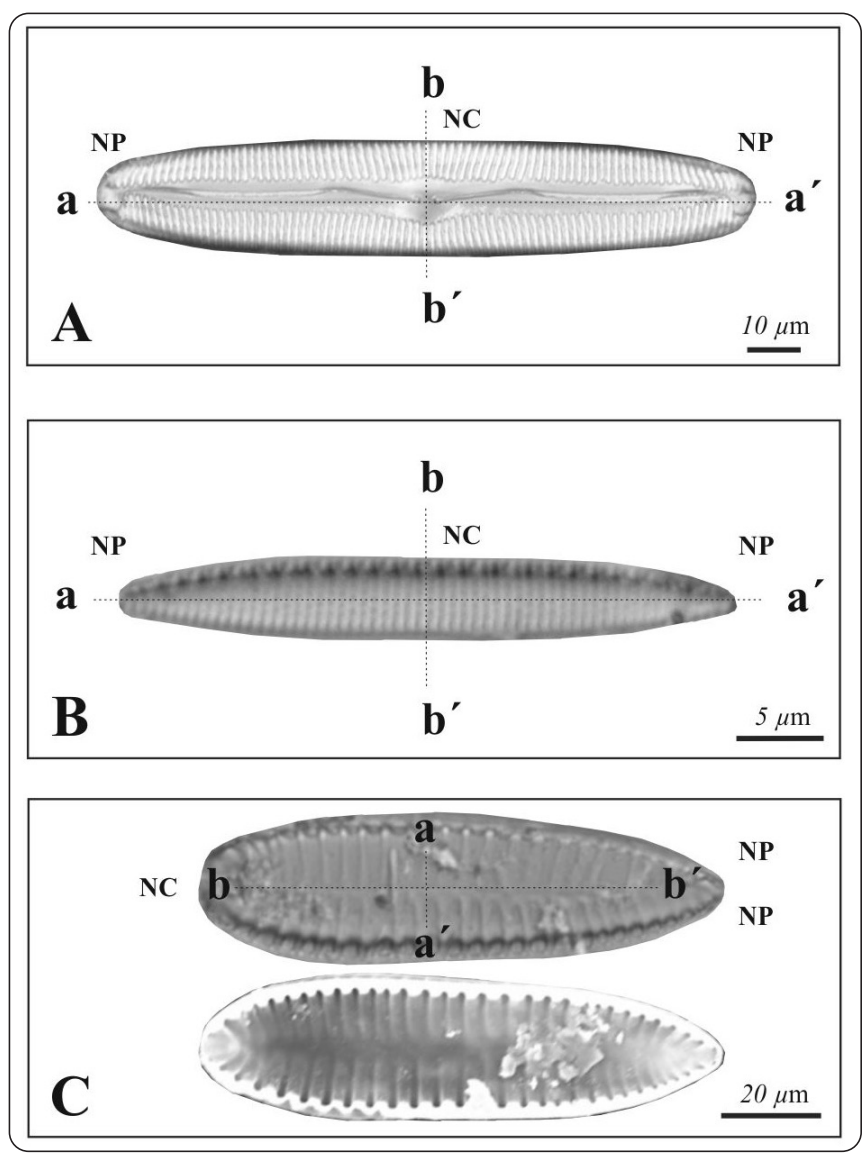

Figura 1. Ilustraciones del sistema del rafe en tres géneros: Pinnularia, Nitzschia e Iconella. Eje apical (a-a'), eje transapical (b-b'), Nódulo polar (NP), Nódulo central (NC). A. Cara valvar lineal, isopolar (Pinnularia viridis), eje apical (a-a') más desarrollado que el transapical (b-b'). B. Valva lineal, isopolar (Nitzschia amphibia) con rafe lateral y canal del rafe elevado en quilla, soportado por fíbulas, eje apical (a-a') más desarrollado que el transapical (b-b'). C. Valva ovalada, heteropolar (Iconella tenera). Rafe circunferencial y canal del rafe elevado en quilla en ambos márgenes formando un ala, eje apical (a-a') menos desarrollado que el eje transapical (b-b'). 
polares (extremos distales) del sistema del rafe (Jahn, Kusber \& Cocquyt, 2017). Se considera que esta disposición es una adaptación para lograr un mayor contacto del sistema del rafe con el sustrato y mejorar la tracción y el desplazamiento, de esta forma la mayoría de las especies en estos géneros son de hábito bentónico, solitario y pueden existir tanto en ambientes salobres como de agua dulce. Estas especies anteriormente estaban incluidas en los géneros Surirella (dividida en dos grupos principales Pinnatae y Robustae, Krammer \& Lange-Bertalot 1987) y Cymatopleura Smith, Stenopterobia Brébisson ex Van Heurck y Campylodiscus del grupo Robusti. En los análisis realizados por Ruck et al. (2016a) se estableció que existen dos clados principales dentro de este grupo de especies. El primero incluye a especies predominantemente salobres y dulceacuícolas del grupo Surirella Pinnatae y el género Cymatopleura, en las que el canal del rafe se localiza sobre la valva y se comunica con el interior celular a través de poros simples (portulae) de diferentes grosores. El segundo grupo incluye a especies predominantemente dulceacuícolas dentro de los grupos Surirella Robustae y Campylodyscus Robusti (Paddock, 1985; Krammer \& Lange-Bertalot, 1987) y el género Stenopterobia, en las que el canal del rafe se encuentra elevado con respecto a la superficie valvar, formando una quilla que conecta con el interior celular por canales alares (fibulae) uniformemente porosos, que se encuentran separados por aperturas (fenestrae). De esta forma, según esta propuesta las especies dentro del primer clado permanecen en el género Surirella, mientras que las del segundo fueron transferidas al género Iconella Jurilj (Ruck, Nakov, Alverson \& Theriot, 2016b).

De acuerdo con esta propuesta, la especie Surirella guatimalensis Ehrenberg ha sido transferida al género Iconella como I. guatimalensis (Ehrenberg) Ruck \& Nakov. Esta es una especie, como ya se mencionó, de gran tamaño dentro de las diatomeas dulceacuícolas por tener una morfología muy especial, ya que las terminaciones proximales del rafe (nódulo central) son muy distintas a las de otras especies dentro de las Surirellales (Ruck \& Theriot, 2011; Ruck et al., 2016a). Esta especie ha sido reportada en varias localidades de distintos países, inicialmente por Ehrenberg (1854) a partir de material colectado en Guatemala y parece tener una distribución preferencial en el Neotrópico (Guerrero, Sala \& Gorriti, 2001; Seeligman, Morales \& Maidana, 2008), también en distintas regiones, en Brasil (Bicudo, Corte Real \& Martau, 1973; Aguiar \& Martau, 1979; Ribeiro, Alves, Moreno \& Santos, 2009; Salomoni \& Torgan, 2010; Morais \& Bicudo, 2017; Morais \& Bicudo, 2018; Pereira et al., 2018), Colombia (Modesto-Iregui, Guillot, Donato \& Otegón, 2002), Argentina (Guerrero et al., 2001; Seeligman et al., 2008), centro y sur de México (Sánchez-Rodríguez \& Vázquez, 1990; Vázquez \& Favila, 1998; Díaz-Pardo, Vázquez \& Guerra-Magaña, 2002; Pedraza, 2020). Estos reportes han sido enfocados principalmente al estudio de la diversidad de las comunidades de algas en lagos, humedales y ríos, ocasionalmente describiendo su abundancia o las condiciones ambientales en que se desarrollan. Por ejemplo, para Brasil se cita como abundante en el río Guamá (Ribeiro et al., 2009) y también reportada en algunas presas con aguas mesotróficas, dulces y levemente ácidas (pH de 6.5, Morais et al., 2018). En Argentina tiene una amplia distribución desde la Patagonia en el sur (Guerrero et al., 2001) hasta Jujuy en el norte del país (Seeligmann et al., 2008). En México, se ha encuentrado en dos lagos: el lago Atezca, Hidalgo, un lago mesotrófico de agua dulce y alcalina (pH 7.3 a 9.3, Vázquez \& Favila, 1998) y en el lago Cantemual, Tabasco, un lago somero de agua dulce y levemente alcalina (pH 7.5 a 8.5, Pedraza, 2020).

En este trabajo reportamos que en México esta especie también está presente en sedimentos del Holoceno provenientes del lago Santa María del Oro, Nayarit y del lago Coatetelco, Morelos y exploramos su potencial como indicador paleoambiental de condiciones lacustres someras. Al mismo tiempo, incluimos una descripción morfológica de la especie apoyada con ilustraciones tanto en Microscopía de Luz (ML) como en Microscopía Electrónica de Barrido (MEB) de los ejemplares revisados.

\section{Materiales y Métodos}

\section{Sitio de estudio}

Los dos sitios de estudio son lagos ubicados a lo largo de la Faja Volcánica Trans-Mexicana (FVTM), el lago Santa María del Oro (SMO) que se ubica en el extremo oeste de la FVTM, a los $21^{\circ} 22^{\prime} \mathrm{N}$ y $104^{\circ} 34^{\prime} \mathrm{O}$, a una elevación de $750 \mathrm{msnm}$ mientras que el lago de Coatetelco (COA) se localiza en el extremo sur de la FVTM a los $18^{\circ} 45^{\prime} \mathrm{N}$ y $99^{\circ} 20^{\prime} \mathrm{O}$, a una elevación de 900 msnm (Figura 2). El lago SMO tiene un área de 370 ha y una profundidad máxima de $65 \mathrm{~m}$. Se encuentra en un clima tropical subhúmedo con lluvias en verano $\left(\mathrm{Aw}_{0}\right)$, con una temperatura media anual de $20.9^{\circ} \mathrm{C}$ y precipitación de $1,220 \mathrm{~mm}$ al año, concentrada entre junio y septiembre. Es un lago mesotrófico, tropical monomíctico (estratifica durante los meses cálidos del año y mezcla o recircula durante los meses fríos), subsalino (con sólidos disueltos totales de $792 \mathrm{mg} \mathrm{L}^{-1}$ ) y alcalino (pH de 8.6) (Caballero et al., 2013; Sigala et al., 2017). El lago COA tiene una superficie de 175 ha y una profundidad máxima de aproximadamente $4 \mathrm{~m}$, también se encuentra en un clima tropical subhúmedo con lluvias en verano, en donde la temperatura media anual es de $23.6{ }^{\circ} \mathrm{C}$ y la precipitación de $1,068 \mathrm{~mm}$ al año, concentrada entre mayo y octubre. Es un lago eutrófico a hipertrófico, polimíctico cálido (mezcla frecuentemente a lo largo del año), de agua dulce (sólidos disueltos totales de $462 \mathrm{mg} \mathrm{L}^{-1}$ ) y alcalina (pH de 8.3) (DíazVargas, Molina-Astudillo, García \& Elizalde-Arriaga, 2017; Avendaño et al., 2021).

\section{Las secuencias sedimentarias}

El material estudiado en este trabajo correspondió a muestras de sedimento provenientes de tres secuencias estratigráficas, dos del lago SMO y una del lago COA. La primera secuencia fue obtenida de una zona litoral del lago SMO (a $12 \mathrm{~m}$ de 


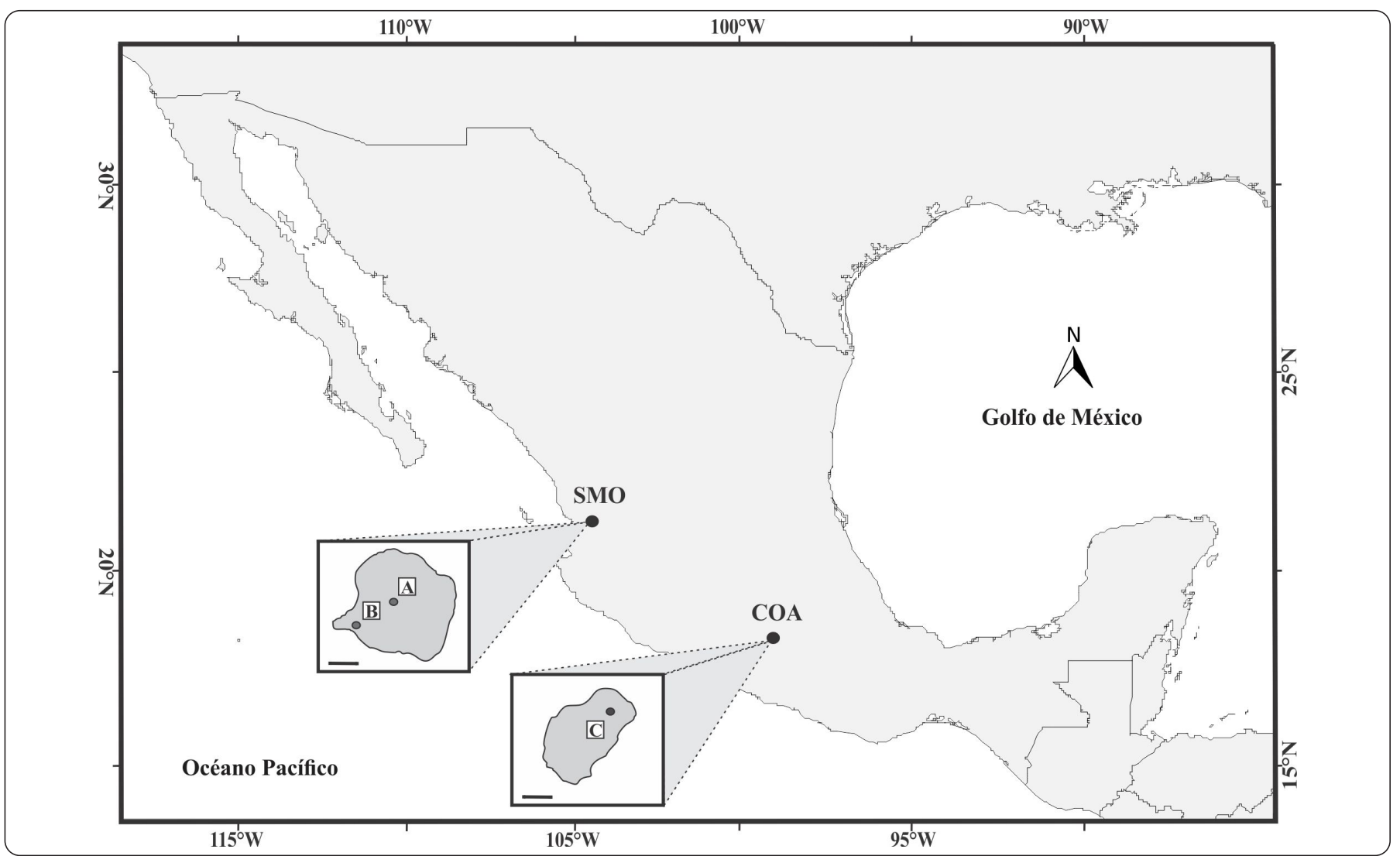

Figura 2. Localización geográfica de los lagos Santa María del Oro (SMO), Nayarit y Coatetelco (COA), Morelos. La escala en los recuadros equivale a 500 m. A. Núcleo MOLESMO03. B. Núcleo SMO02V. C. Núcleo COA15.

profundidad), denominada SMO02V, tiene $8.8 \mathrm{~m}$ de profundidad y cuenta con un modelo de edad basado en 7 dataciones por radiocarbono calibradas (Vázquez-Castro, Ortega-Guerrero, Rodríguez, Caballero \& Lozano-García, 2008; RodríguezRamírez et al., 2015). Este modelo de edad permite establecer que la base de esta secuencia data de hace 2,500 años antes del presente (A.P.). La segunda secuencia recuperada del lago SMO proviene de la zona central (a $57 \mathrm{~m}$ de profundidad), se denomina MOLESMO03 y tiene $8.9 \mathrm{~m}$ de profundidad. Para esta secuencia también se cuenta con un modelo de edad basado en dataciones radiométricas calibradas (Lozano et al., 2020) que indican que la base de esta secuencia data de hace 5,000 años cal. A.P. En el lago COA se recuperó una secuencia sedimentaria en su zona central, esta secuencia se denomina COA15 y tiene $2.6 \mathrm{~m}$ de longitud. El modelo de edad para esta secuencia se basa en tres dataciones por radiocarbono calibradas (Vázquez, 2021) y establece que la base de la secuencia abarca los últimos 2,100 años cal. A.P.

\section{Análisis de las diatomeas}

Los análisis de las diatomeas se realizaron usando $0.5 \mathrm{~g}$ de sedimento seco, que fue procesado sucesivamente con ácido clorhídrico al 10\%, para la eliminación de carbonatos, pirofosfato de sodio al 10\% para dispersar el sedimento, peróxido de hidrógeno concentrado $(30 \%)$ y ácido nítrico concentrado $(70 \%)$ para la eliminación de materia orgánica. Una vez procesadas las muestras se elaboraron preparaciones permanentes, que fueron montadas con resina Naphrax. El gran tamaño de las valvas de I. guatimalensis puede representar un problema metodológico para la determinación de su abundancia real en las muestras, ya que pueden quedar excluidas de las alícuotas con las que se elaboran las preparaciones para el ML, por lo que es conveniente que cuando se trabaje con muestras que puedan tener diatomeas de gran tamaño se tomen medidas que aseguren un submuestreo adecuado (Lavoie, Campeau, Fallu \& Dillon, 2006). Esto puede incluir medidas tan sencillas como el despuntado de las puntas para micropipeta con las que se coleta la alícuota para el montaje de las preparaciones permanentes.

La identificación de las especies, la toma de fotografías y la medición de las valvas se realizó bajo el MLa 1000x utilizando un Olympus BX50 y un Axioscope Zeiss. Para la observación al MEB (JEOLJCM-6000 Neoscope) se elaboraron preparaciones sobre placas de aluminio que se recubrieron con oro. Por último, se realizaron conteos mínimos de 100 valvas de diatomeas por muestra, en los que se estimaron las abundancias relativas (\%) de las especies identificadas. Las muestras que se incluyen en 
este trabajo fueron aquellas en las que se observó la presencia de I. guatimalensis. Adicionalmente, se incluyen datos del análisis de diatomeas de la secuencia MOLESMO03, presentando la sumatoria de todas las especies con forma de vida bentónicoperifítico, esto con el objetivo de correlacionar la presencia de I. guatimalensis contra la abundancia de las diatomeas con esta forma de vida, que domina en ambientes lacustres someros.

\section{RESULTADOS}

En las Figuras 3 (a-e) y 4 (a-e) se ilustran algunos de los ejemplares de I. guatimalensis provenientes de las tres secuencias sedimentarias y en la Tabla I se presentan los principales parámetros morfológicos de los ejemplares medidos. Los ejemplares de la especie estuvieron presentes con baja abundancia en 17 muestras de sedimento del núcleo central del lago SMO (MOLESMO03, <1\%), en 21 muestras del núcleo litoral de este mismo lago (SMO02V, <10\%) y en 3 del núcleo central del lago COA (COA15, <2\%).

En la Figura 5 se ilustran las abundancias relativas (\%) de esta especie a lo largo del tiempo en las tres secuencias sedimentarias
(MOLESMO03, SMO02V y COA15). Se puede observar que la distribución a lo largo del tiempo de esta especie en los tres sitios se concentra en cuatro ventanas temporales: I) entre los años 3100 a 2300 a.C. (5050-4260 años cal. A.P.); II) entre los 1040 a 580 a.C. (3000-2530 años cal. A.P.); III) a los 50-900 d.C. (2000-1050 años cal. A.P.); y IV) entre los años 1315-1860 d.C. (635-90 años cal. A.P.). En la Figura 6 se presenta la abundancia de I. guatimalensis contrastada contra la sumatoria de las especies de hábito bentónico-perifítico (Tabla II) de la secuencia sedimentaria MOLESMO03, en donde se observa una buena coincidencia entre distribución de I. guatimalensis con el resto de las especies indicadoras de ambientes someros.

\section{Descripción morfológica de la especie}

Iconella guatimalensis (Ehrenberg) Ruck \& Nakov 2016. Basiónimo: Surirella guatimalensis Ehrenberg 1854

La cara valvar es oval, heteropolar (Figuras 3 a-e y Figuras $4 a$ y $4 \mathrm{e}$ ), con el polo anterior ampliamente redondeado, mientras que el polo basal es agudo. Los ejemplares observados en el lago SMO tuvieron un rango de tamaños entre 124 a $160 \mu \mathrm{m}$

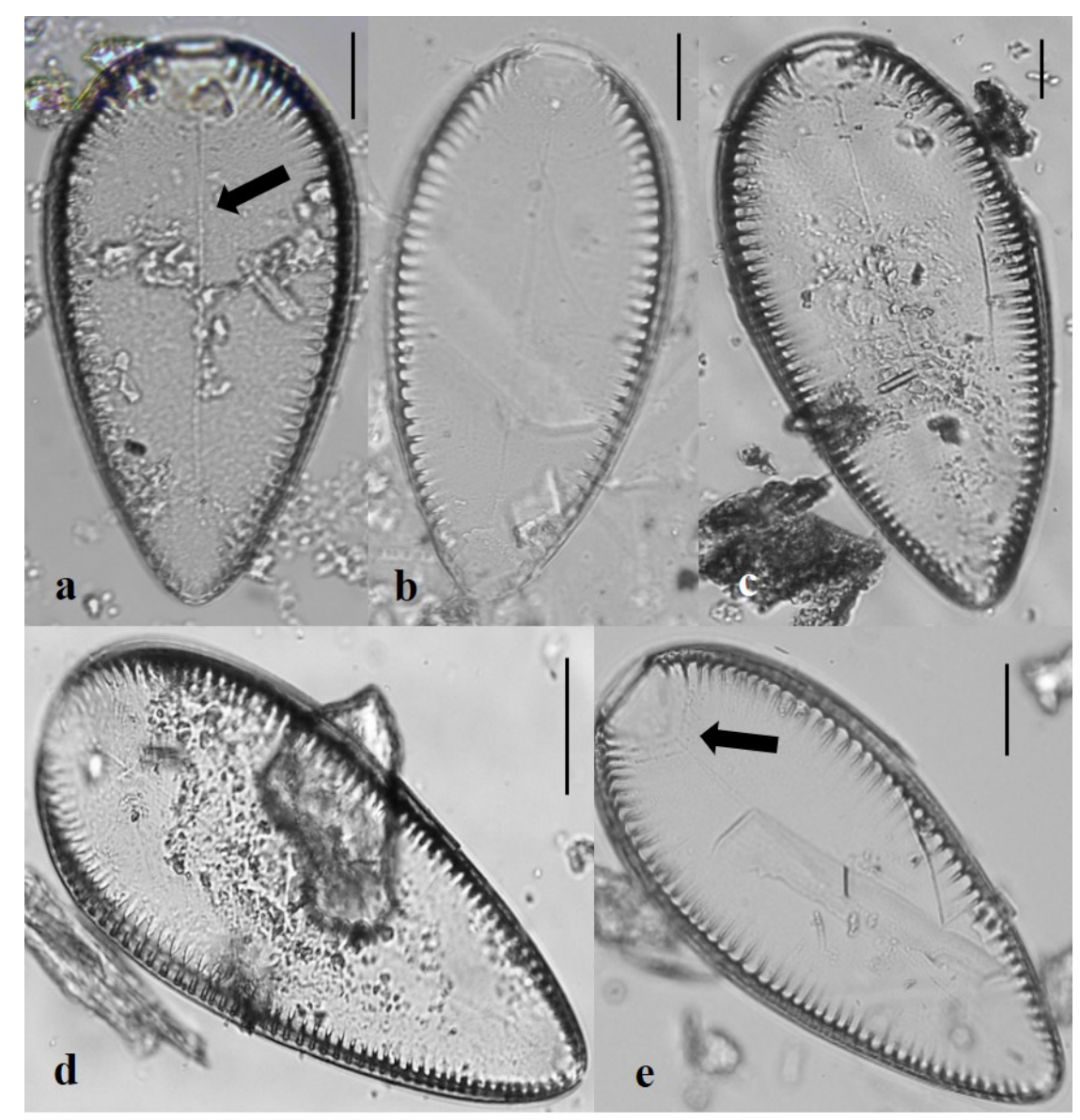

Figura 3. Iconella guatimalensis. Microscopio de luz (100x). Escala de la barra $20 \mu \mathrm{m}$. a-e. Vista valvar. a. La flecha indica la línea axial. e. La flecha indica la región cóncava en el polo anterior con la terminación proximal del rafe. 


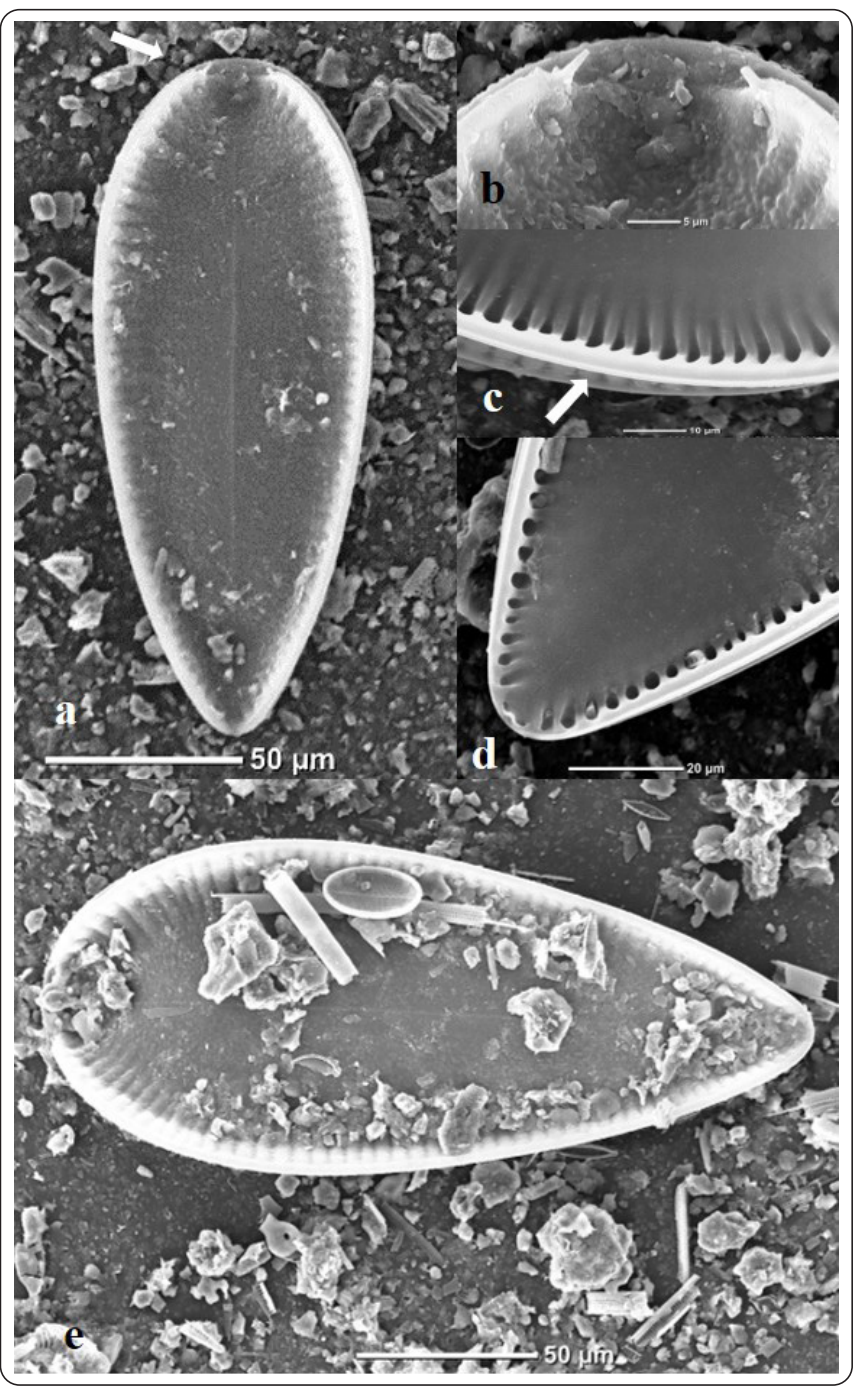

Figura 4. Iconella guatimalensis. Microscopio electrónico de barrido. a. Vista valvar $(450 \mathrm{X})$. b. Terminación proximal del rafe en el polo anterior $(3,400 \mathrm{X})$. c. Detalle de las fíbulas $(2,000 \mathrm{X})$. La flecha indica el canal del rafe. d. Terminación del polo basal de la valva $(1,500 \mathrm{X})$. e. Vista valvar que ofrece un comparativo de las dimensiones de I. guatimalensis y otras especies como Aulacoseira granulata y Cocconeis placentula $(500 \mathrm{X})$.

de largo, un ancho valvar entre 52 a $94 \mu \mathrm{m}$ y una densidad de fibulae de 26 a 30 en $100 \mu \mathrm{m}$. En el caso de los ejemplares de COA, éstos tuvieron tamaños similares, con un rango de 107 a $195 \mu \mathrm{m}$ de largo, 41 a $85 \mu \mathrm{m}$ de ancho valvar, pero tuvieron fibulae con un rango de densidades más elevado, entre 40 a 60 en $100 \mu \mathrm{m}$ (Tabla I).

La cara valvar presenta una característica línea media formada por un ligero engrosamiento (cresta) de la valva a lo largo del eje transapical (Figura 3a). En la parte anterior, redondeada de la valva, existe una pequeña región cóncava con un adelgazamiento en donde convergen dos proyecciones que representan las
Tabla I. Parámetros morfológicos de I. guatimalensis de acuerdo con las mediciones de los ejemplares analizados en los sitios de estudio Santa María del Oro (MOLEMO3 y SMO2V) y Coatetelco (COA15) en el centro de México.

\begin{tabular}{|l|c|c|c|}
\hline $\begin{array}{c}\text { Secuencia y } \\
\text { número de valvas } \\
\text { medidas }\end{array}$ & $\begin{array}{c}\text { Longitud } \\
\text { de la valva } \\
(\boldsymbol{\mu} \mathbf{m})\end{array}$ & $\begin{array}{c}\text { Ancho de } \\
\text { la valva } \\
(\boldsymbol{\mu} \mathbf{m})\end{array}$ & $\begin{array}{c}\text { Densidad de } \\
\text { fíbulas (en } \\
\mathbf{1 0 0} \boldsymbol{\mu} \mathbf{m})\end{array}$ \\
\hline MOLESMO03 (6) & $124-145$ & $52-94$ & 30 \\
\hline SMO02V (5) & $130-160$ & $61-78$ & $26-28$ \\
\hline COA15 (3) & $107-195$ & $41-85$ & $40-60$ \\
\hline
\end{tabular}

Tabla II. Listado de las especies incluidas en la categoría bentónicasperifíticas de la secuencia sedimentaria MOLESMO03, proveniente del lago Santa María del Oro, Nayarit, centro de México.

\begin{tabular}{|c|}
\hline Achnanthidium exiguum (Grunow) Czarnecki 1994 \\
\hline Amphora copulata (Kützing) Schoeman \& Archibald 1986 \\
\hline Anomoeoneis sphaerophora Pfitzer 1871 \\
\hline Campylodiscus hibernicus Ehrenberg 1845 \\
\hline Cocconeis placentula Ehrenberg 1838 \\
\hline Cymbella mexicana (Ehrenberg) Cleve 1894 \\
\hline Epithemia adnata (Kützing) Brébisson 1938 \\
\hline Epithemia sorex Kützing 1944 \\
\hline Fragilaria capucina Desmazières 1830 \\
\hline Gomphonema affine Kützing 1844 \\
\hline Gomphonema parvulum (Kützing) Kützing 1849 \\
\hline Halamphora coffeaeformis (C. Agardh) Levkov 2009 \\
\hline Halamphora veneta (C. Agardh) Levkov 2009 \\
\hline $\begin{array}{l}\text { Hantzschia amphioxys (Ehrenberg) Grunow in Cleve \& } \\
\text { Grunow } 1880\end{array}$ \\
\hline $\begin{array}{l}\text { Hippodonta hungarica (Grunow) Lange-Bertalot, Metzeltin } \\
\text { \& Witkowski } 1996\end{array}$ \\
\hline Iconella tenera (W. Gregory) Ruck \& Nakov 2016 \\
\hline Luticola mutica Kützing R.M. Crawford \& D.G. Mann 1990 \\
\hline Pinnularia viridis (Nitzsch) Ehrenberg 1843 \\
\hline $\begin{array}{l}\text { Planothidium delicatulum (Kützing) Round \& Bukhtiyarova } \\
1996\end{array}$ \\
\hline Rhoicosphenia abbreviata (C. Agardh) Lange-Bertalot 1980 \\
\hline $\begin{array}{l}\text { Rhopalodia acuminata Krammer in Lange-Bertalot \& } \\
\text { Krammer } 1987\end{array}$ \\
\hline Rhopalodia gibba (Kützing) O. Müller 1895 \\
\hline Rhopalodia gibberula (Ehrenberg) O. Müller 1899 \\
\hline Sellaphora laevissima (Kützing) Mann 1989 \\
\hline Sellaphora pupula (Kützing) Mereschkovsky 1902 \\
\hline Terpsinoë música Ehrenberg 1843 \\
\hline Tryblionella granulata (Grunow) D.G. Mann (1990) \\
\hline
\end{tabular}




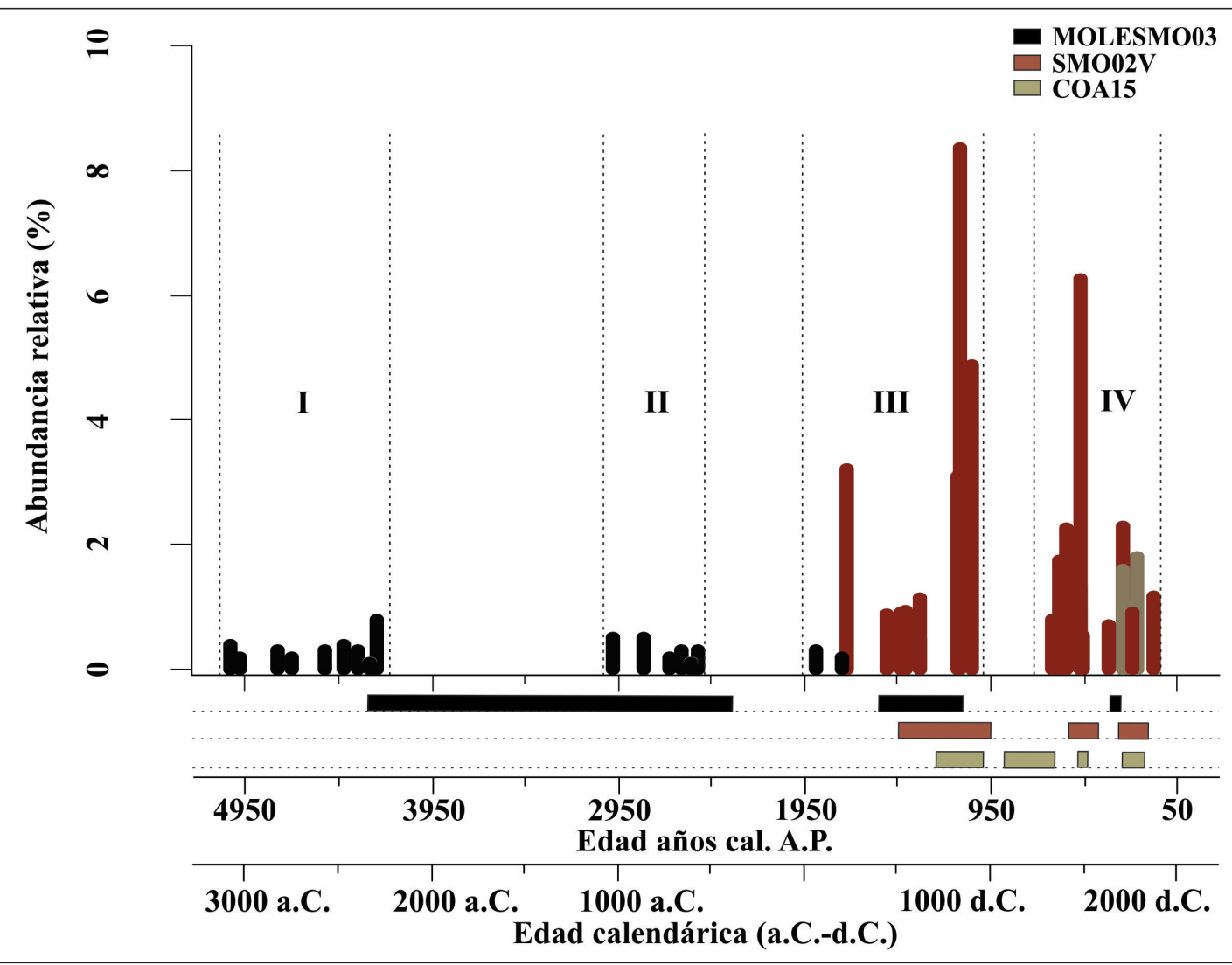

Figura 5. Abundancia relativa (\%) de Iconella guatimalensis durante los últimos 5000 años en las secuencias sedimentarias del lago Santa María del Oro (MOLESMO03 y SMO02V) y del lago Coatetelco (COA15). Los números romanos (I, II, III y IV) identifican las ventanas temporales en las que se registra la presencia de I. guatimalensis en las secuencias estratigráficas analizadas. Las barras en la parte inferior de la figura destacan las secciones de cada una de las secuencias estratigráficas en las que se interpretan condiciones de sequía con base en otros indicadores paleoambientales.

terminaciones proximales del rafe (Figuras $3 \mathrm{e}$ y $4 \mathrm{~b}$ ). El rafe es circunferencial y se ubica en un canal elevado (ala o quilla) poco desarrollado (Figura 4c). Los extremos distales del rafe, por su parte, se ubican en la parte basal aguda de la valva. Las fibulae conforman los soportes internos del ala que contiene al rafe, son cortos y no llegan a la zona central (Figuras 4c y 4d). Los canales alares tienen aberturas fenestrales ocluidas (Jahn, Kusber \& Cocquyt, 2017). Las estrías son inconspicuas o escasamente visibles al ML (Guerrero et al., 2001; Morais \& Bicudo, 2017).

\section{Discusión}

En este trabajo los ejemplares de I. guatimalensis tuvieron un rango de tamaño intermedio en la longitud de la valva (107$195 \mu \mathrm{m})$ con respecto al rango bibliográfico reportado que va entre 124-261 $\mu \mathrm{m}$ (Guerrero et al., 2001; Seeligmann et al., 2008; Salomoni \& Torgan, 2010; Morais et al., 2018; Pedraza, 2020). Por su parte la densidad de las fibulae (canales alares) fue de 26-60 en $100 \mu \mathrm{m}$, con los valores más elevados registrados para los ejemplares del lago COA. Estos rangos caen por arriba del dato bibliográfico reportado que va entre 20-30 en $100 \mu \mathrm{m}$ y aunque algunos autores como Morais et al. (2018) mencionan que pueden llegar hasta $60-80$ en $100 \mu \mathrm{m}$, los valores son muy similares a los encontrados en los ejemplares de COA.

Iconella guatimalensis es una diatomea que no está presente actualmente en los sedimentos de SMO o de COA, sin embargo, esta especie sí lo está en las secuencias sedimentarias del Holoceno de los dos lagos estudiados (SMO y COA) en una baja abundancia $(<10 \%)$. Sin embargo, por el gran tamaño celular de esta especie y su baja abundancia relativa puede dar una impresión poco precisa de su importancia en el registro sedimentario de estos lagos, al considerar que por su gran tamaño tiene una biomasa mayor que la de otras especies de menor talla (Lavoie et al., 2006). Este es un problema de representatividad en las comunidades de microrganismos que ha sido comúnmente abordado mediante la transformación de los datos de abundancia relativa a biovolumen, una aproximación muy común en estudios de fitoplancton (Sun \& Liu, 2003) pero que ha sido poco empleada en los análisis de diatomeas en sedimentos (Caballero, Vázquez, Ortega, Favila \& LozanoGarcía, 2016). 


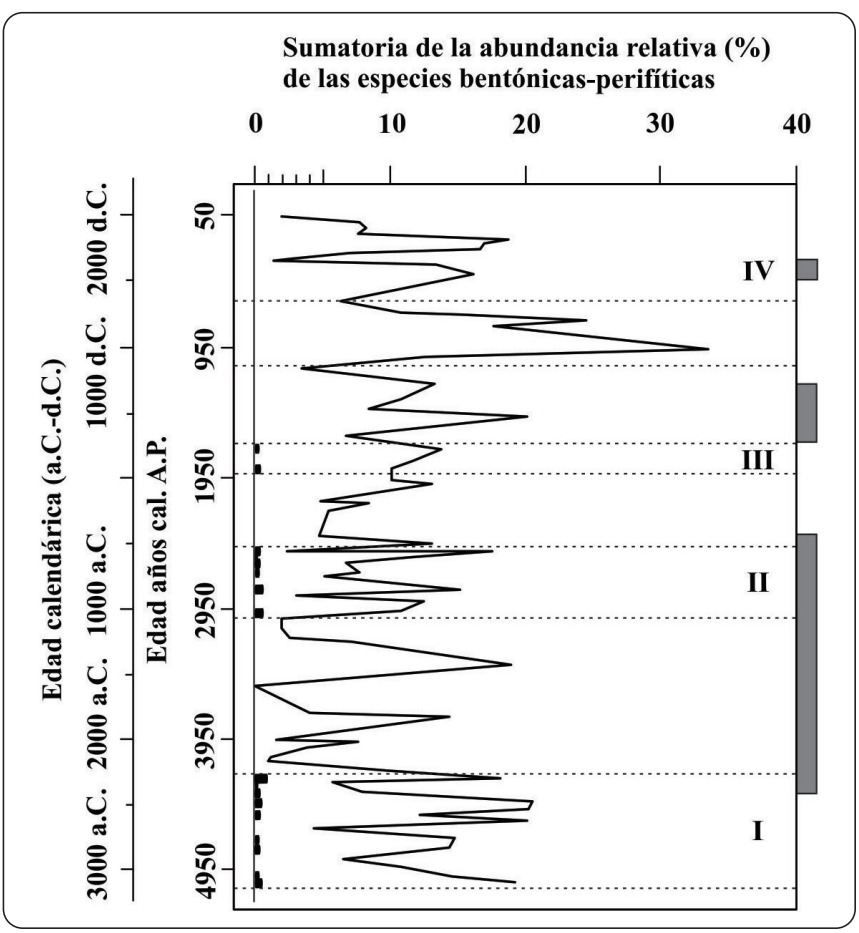

Figura 6. Sumatoria de la abundancia relativa (\%) de las diatomeas de hábito bentónico-perifítico, abundancia relativa de Iconella guatimalensis, y ventas de tiempo para las cuales se infieren condiciones de sequía con base en diversos proxis paleoambientales (Lozano et al., 2020) a lo largo del tiempo en la secuencia sedimentaria MOLESMO03 del lago Santa María del Oro, Nayarit.

La distribución de I. guatimalensis en la secuencia MOLESMO03 coincide con momentos en que las diatomeas de hábito bentónico-perifítico tienen una mayor abundancia, como lo muestra la Figura 6, aunque también es cierto que no en todas las ventanas de tiempo en las que las especies bentónico-perifítico son abundantes se registra la presencia de I. guatimalensis. Esto sugiere que, a pesar de tener poca información sobre la distribución ecológica de esta especie, es posible considerar que I. guatimalensis es parte de la comunidad de diatomeas bentónicas (Vázquez \& Favila, 1998; Pedraza, 2020), de acuerdo con lo esperado para una especie con rafe periférico, como es característico del orden Surirellales, y que se considera una adaptación que favorece el desplazamiento sobre el sedimento en el ambiente bentónico.

De esta forma, su distribución en las cuatro ventanas de tiempo, identificadas a lo largo de los últimos 5000 años A.P., representan eventos de nivel lacustre bajo en SMO y COA (Figura 5). Estas ventanas corresponden a los años I) 3100 a 2300 a.C. (5050-4260 años cal. A.P.); II) 1040 a 580 a.C., III) 50-900 d.C. y IV) 1,315-1860 d.C. Elúltimo intervalo registrado (IV) es posible subdividirlo en dos momentos, entre 1315 a 1560 d.C. y entre 1600 a 1860 d.C. Estas cuatro ventanas de tiempo coinciden de forma cercana con los lapsos en los que se han identificado ambientes someros para el lago SMO con base en otros indicadores paleoambientales como el polen o la geoquímica (Rodríguez-Ramírez et al., 2015; Lozano et al., 2020). En la secuencia sedimentaria MOLESMO03 estudios de geoquímica y polen han permitido identificar tres eventos de condiciones de sequía durante los últimos 5000 años del Holoceno (Lozano et al., 2020) (Figura 5), en particular dos de ellos que corresponden muy bien con los incrementos más recientes en I. guatimalensis aquí reportados (III y IV). Estos dos eventos se ubican entre 380 a 850 d.C. y alrededor del año 1650 d.C. Por otra parte, en el núcleo SMO02V se han realizado estudios de diatomeas, ostrácodos, mineralogía magnética y geoquímica que indican tres eventos de nivel lacustre bajo y condiciones de relativa sequía durante los últimos 2000 años cal. A.P. (Vázquez-Castro et al., 2008; Rodríguez-Ramírez et al., 2015), que también correlacionan con los incrementos de I. guatimalensis (III, IV), estos eventos datan entre los 500 y 1000 d.C., entre 1400 a 1550 d.C. y el último entre 1690 a 1770 d.C. (Figura 5). En la secuencia COA15 se han realizado estudios de diatomeas y geoquímica (Vázquez, 2021) que ubican cuatro momentos de condiciones de sequía en los últimos 2000 años que nuevamente correlacionan las ventanas de tiempo (III, IV) en las que hubo incrementos de I. guatimalensis, el primero, entre 700 a 950 d.C.; el segundo aproximadamente de $\operatorname{los} 1100$ a 1350 d.C.; el tercero alrededor del año 1500 d.C.; y elúltimo entre 1700 a 1800 d.C. (Figura 5).

En particular, el incremento de I. guatimalensis detectado entre 50 y 900 d.C. (III), es un momento ampliamente reconocido en el Centro de México como una etapa de condiciones climáticas dominadas por sequías recurrentes que tuvieron su mayor impacto entre los años 600 a 1000 d.C. Este intervalo de sequías recurrentes se conoce como las Sequías del Clásico Tardío, y han sido asociadas con el abandono y colapso cultural de diversas ciudades tanto en la zona Maya como a lo largo de toda Mesoamérica (Hodell, Brenner \& Curtis, 2005; RodríguezRamírez et al., 2015). Por su parte, el intervalo en el que se incrementa I. guatimalensis es en dos eventos: entre 1315 a 1560 d.C. y entre 1600 a 1860 d.C. (IV) correspondientes con la llamada Pequeña Edad de Hielo que en muchos registros de México se ha identificado con condiciones frías y secas en dos etapas de mayor intensidad, entre aproximadamente 1400 a 1550 d.C. y entre 1650 a 1800 d.C.

\section{Conclusiones}

Se identifican cuatro eventos durante los últimos 5000 años en los que se registran mayores-abundancias de I. guatimalensis en los sedimentos de los lagos SMO y COA. Estas ventanas de tiempo corresponden con períodos en que otros indicadores paleoambientales también apuntan a condiciones de sequía y niveles lacustres bajos en SMO y en COA, corroborando la hipótesis de que I. guatimalensis es un buen indicador paleolimnológico de condiciones lacustres someras. 


\section{Agradecimientos}

Al Posgrado en Ciencias del Mar y Limnología, UNAM por las facilidades otorgadas para la elaboración de este trabajo. Al Consejo Nacional de Ciencia y Tecnología (CONACYT) por la beca otorgada (41357) al primer autor para la realización de la presente investigación. A la M. en C. Laura Gómez del Servicio Académico de Microscopía Electrónica de Barrido (SAMEB), Instituto de Ciencias del Mar y Limnología-UNAM por su apoyo para el recubrimiento de muestras de diatomeas, y a la Dra. Socorro Lozano y la Dra. Susana Sousa del laboratorio de Paleoecología, Paleoclimatología y Cambio climático, Instituto de Geología-UNAM por su apoyo en la realización de las micrografías al MEB.

\section{REFERENCIAS}

Aguiar, L. W. \& Martau, L. (1979). Diatomáceas de lagos do Parque Zoologico, Rio Grande do Sul, Brasil. Iheringia, Série Botânica, 25, 27-110.

Avendaño, D., Caballero, M. \& Vázquez, G. (2021). Ecological distribution of Stephanodiscus niagarae Ehrenberg in central Mexico and niche modeling for its last glacial maximum habitat suitability in the Nearctic realm. Journal of Paleolimnology, 66, 1-14. DOI: 10.1007/. s10933-02100178-w

Bicudo, C.E.M., Corte Real, M. \& Martau, L. (1973). Catálogo das algas de águas continentais do Estado do Rio Grande do sul, Brasil: I. Bacillariophyceae. Iheringia, Série Botânica, 17, 56-68.

Caballero, M., Rodríguez, A., Vilaclara, G., Ortega, B., Roy, P. \& Lozano García, S. (2013). Hydrochemistry, ostracods and diatoms in a deep, tropical, crater lake in Western Mexico. Journal of Limnology, 72 (3), 512-523. DOI: 10.4081/ jlimnol.2013.e42

Caballero, M., Vázquez, G., Ortega, B., Favila, M. E. \& Lozano-García, S. (2016). Responses to a warming trend and "El Niño" events in a tropical lake in western México. Aquatic Sciences, 78(3), 591-604. DOI: 10.1007/s00027015-0444-1

Caballero, M., Lozano-García, S., Ortega-Guerrero, D. \& Correa-Metrio,A. (2019). Quantitative estimates of orbital and millenial scale climatic variability in central Mexico during the las 40,000 years. Quaternary Science Reviews, 205, 62-75. DOI:10.1016/j.quascirev.2018.12.002

Díaz-Pardo, E., Vázquez, G. \& Guerra-Magaña, C. (2002). Lago Atezca. En G. De la Lanza Espino \& J. L. García Calderón (Eds.). Lagos y Presas de México (pp. 109-126). México: AGT Editor, S.A.

Díaz-Vargas, M., Molina-Astudillo, F., García, J. \& ElizaldeArriaga, E. (2017). Estado trófico del lago de Coatetelco, Morelos, México. Investigación Agropecuaria, 14(3), 145-152.

Ehrenberg, C. (1854). Mikrogeologie. Einundvierzig Tafeln mit ûber viertausend frossentheils colorirten Figuren, Gzeichnet vom Verfasser.
Guerrero, J., Sala, S. \& Gorriti, G. (2001). Epithemiaceae y Surirellaceae (Bacillariophyceae) de Tierra del Fuego, Argentina. Boletín de la Sociedad Botánica Argentina, 36(12), 29-45. Recuperado a partir de https://botanicaargentina. org.ar/wp-content/uploads/2018/06/29-45.pdf

Hodell, D., Brenner, M. \& Curtis, J. (2005). Terminal Classic drought in the northern Maya lowlands inferred from multiple sediment cores in Lake Chichancanab (México). Quaternary Science Reviews, 24, 1413-1427. DOI: 10.1016/j.quascirev.2004.10.013

Jahn, R., Kusber, W. \& Cocquyt, C. (2017). Differentiating Iconella from Surirella (Bacillariophyceae): typifying four Ehrenberg names and a preliminary checklist of the African taxa. PhytoKeys, 82, 73-112. DOI: 10.3897/ phytokeys.82.13542

Krammer, K., \& Lange-Bertalot, H. (1987). Morphology and taxonomy of Surirella ovalis and related taxa. Diatom Research, 2(1), 77-95.

Lavoie, I., Campeau, S., Fallu, M. \& Dillon, P. (2006). Diatoms and biomonitoring: Should cell size be accounted for? Hydrobiologia. 573, 1-16. DOI: 10.1007/s10750-0060223-z

Lozano-García, S., Figueroa-Rangel, B., Sosa-Nájera, S., Caballero, M., Noren, A., Metcalfe, S., Tellez-Valdés, O. \& Ortega-Guerrero, B. (2020). Climatic and anthropogenic influences on vegetation changes during the last 5000 years in a seasonal dry tropical forest at the northern limits of the Neotropics. The Holocene, 31(5) 802-813. DOI: $10.1177 / 0959683620988054$

Modesto-Iregui, M., Guillot, G., Donato, J. \& Otegón, M.(2002). Dimensión fractal y relación área superficial/volumen de algas del fitoplancton de lagos colombianos. Caldasia, 24(1), 121-134. DOI: https://revistas.unal.edu.co/index. $\mathrm{php} / \mathrm{cal} /$ article/view/39437

Morais, K. \& Bicudo, C. (2017). Criptogams del Parque Estatal Fontes do Ipiranga, Sao Paulo, SP. Algas, 42: Bacillariophyceae (Surirellales). Hoehnea, 44(1), 10-28. DOI: 10.1590/2236-8906-26/2018

Morais, K., Rodrigues, E., Zorzal-Almeida, S., Bicudo, D. \& Matos, C. (2018). Taxonomy and ecology of order Surirellales (Bacillariophyceae) in tropical reservoirs in Southeastern of Brazil. Acta Limnologica Brasiliensia, 30, e204. DOI: 10.1590/s2179-975x9817

Paddock, T. B. (1985). Observations and comments on the diatoms Surirella fastuosa and Campylodiscus fastuosus and on other species of similar appearance. Nova Hedwigia, 41(1-4), 417-444.

Pedraza, I. (2020). Relación de la comunidad fitoplanctónica y los factores abióticos del Lago Cantemualen Tabasco. Tesis de Maestría, Instituto de Ciencias del Mar y Limnología, UNAM, 137 págs.

Pereira, G., Pereira, S., Siqueira, L., Costa, R., Fonseca, L., Lamonica-Freire, E., Figueiredo, D. \& Teixeira, M. (2018). Inventory of cyanobacteria and microalgae cited for the 
National Park of the Chapada dos Guimarães (MT) and 80 new record from the region. Biota Neotropica, 18(2), 1-15. DOI: 10.1590/1676-0611-bn-2017-0399

Ribeiro, M., Alves, N., Moreno, M. \& Santos, R. (2009). Composição e distribuição do microfitoplâncton do rio Guamá no trecho entre Belém e São Miguel do Guamá, Pará, Brasil. Boletim do Museu Paraense Emílio Goeldi Ciências Naturais, 4(3), 341-351.

Rodríguez-Ramírez, A., Caballero, M., Roy, P., Ortega, B., Vázquez-Castro, G. \& Lozano-García, S. (2015). Climatic variability and human impact during the last 2000 years in western Mesoamerica: evidence of late Classic (AD 600-900) and Little Ice Age drought events. Climate of the Past, 11, 1239-1248. DOI: 10.5194/cp11-1239-2015

Round, F. E., Crawford, R. M. \& Mann, D. G. (1990). The Diatoms. Biology \& Morphology of the genera. Cambridge University Press.

Ruck, E. \& Theriot, E. (2011). Origin and Evolution of the Canal Raphe System in Diatoms. Protist, 162, 723-737. DOI: $10.1016 /$ j.protis.2011.02.003

Ruck, E., Nakov, T., Alverson, A. \& Theriot, E. (2016a). Phylogeny, ecology, morphological evolution and reclassification of the diatom orders Surirellales and Rhopalodiales. Molecular Phylogenetics and Evolution, 103, 155-171. DOI: 10.1016/j.ympev.2016.07.023

Ruck, E., Nakov, T., Alverson, A. \& Theriot, E. (2016b). Nomenclatural transfers associated with the phylogenetic reclassification of the Surirellales and Rhopalodiales. Notulae Algarum, 10, 1-4. DOI: https://www.researchgate. net/publication/306254354

Sánchez-Rodríguez, M. E. \& Vázquez, G. (1990). Estudio bioecológico de la Laguna Atezca, Hidalgo, México. II. Análisis de la comunidad fitoplanctónica. Anales de la Escuela Nacional de Ciencias Biológicas, Instituto Politécnico Nacional, México, 33, 9-19.
Salomoni, S. \& Torgan, L. (2010). The genus Surirella Turpin (Surirellaceae, Bacillariophyta) in aquatic environment from Delta do Jacuí, State Park, southern of Brazil. Iheringia, Série Botânica, 65(2), 281-290.

Sapelko, T., Pozdnyakov, S., Kuznetsov, D. \& Ludikova, A. (2019). Holocene sedimentation in the central part of Lake Ladoga. Quaternary International, 524, 67-75. DOI: 524. 10.1016/j.quaint.2019.05.028

Seeligmann, C., Morales, M. \& Maidana, N. (2008). Diatomeas (Bacillariophyceae) de Humedales de Altura de La Provincia de Jujuy-Argentina. Boletín de la Sociedad Argentina de Botánica, 43(1-2), 1-17.

Sigala, I., Caballero, M., Correa-Metrio, A., Lozano-García, S., Vázquez, G., Pérez, L. \& Zawisza, E. (2017). Basic limnology of 30 continental waterbodies of the Transmexican Volcanic Belt across climatic and environmental gradients. Boletín de la Sociedad Geológica Mexicana, 69 (2), 310-370. DOI: 10.18268/bsgm2017v69n2a3

Sun, J. \& Liu, D. (2003). Geometric models for calculating cell biovolume and surface area for phytoplankton. Journal of plankton research, 25(11), 1331-1346. DOI: 10.1093/ plankt/fbg096

Vázquez, G. \& Favila, M. (1998). Status of the health conditions of subtropical Atezca Lake. Aquatic Ecosystem Health \& Management,1, 245-255. DOI: 10.1080/14634989808656921

Vázquez-Castro, G., Ortega-Guerrero, B., Rodríguez, A., Caballero, M. \& Lozano-García, S. (2008). Mineralogía magnética como indicador de sequía en los sedimentos lacustres de los últimos ca. 2,600 años de Santa María del Oro, occidente de México. Revista Mexicana de Ciencias Geológicas, 25 (1), 21-38.

Vázquez, M. (2021). En revisión. Asociación ecológica de diatomeas en lago de Coatetelco Morelos, para identificar las variaciones climáticas en el Holoceno tardio. Tesis de Licenciatura, Facultad de Ciencias, UNAM. 68 págs. 